\title{
Deep Green And Monolithic White LEDs Based On Combination Of Short-Period InGaN/ GaN Superlattice And InGaN QWs
}

A. F. Tsatsulnikov, W. V. Lundin, A. V. Sakharov, E. E. Zavarin, S. O. Usov, A. E. Nikolaev, N. V. Kryzhanovskaya, A. E. Chernyakov, A. L. Zakgeim, N. A. Cherkashin, and M. Hytch

Citation: AIP Conference Proceedings 1399, 253 (2011); doi: 10.1063/1.3666350

View online: https://doi.org/10.1063/1.3666350

View Table of Contents: http://aip.scitation.org/toc/apc/1399/1

Published by the American Institute of Physics

\section{Articles you may be interested in}

Optimization of InGaN/GaN superlattice structures for high-efficiency vertical blue light-emitting diodes Journal of Applied Physics 114, 173101 (2013); 10.1063/1.4828488

Carrier localization in the vicinity of dislocations in InGaN

Journal of Applied Physics 121, 013104 (2017); 10.1063/1.4973278

GaN-based light emitting diodes using p-type trench structure for improving internal quantum efficiency Applied Physics Letters 110, 021115 (2017); 10.1063/1.4973995

Spontaneous emission of localized excitons in InGaN single and multiquantum well structures Applied Physics Letters 69, 4188 (1996); 10.1063/1.116981

"Blue" temperature-induced shift and band-tail emission in InGaN-based light sources Applied Physics Letters 71, 569 (1997); 10.1063/1.119797

High-brightness InGaN/AIGaN double-heterostructure blue-green-light-emitting diodes Journal of Applied Physics 76, 8189 (1994); 10.1063/1.357872 


\title{
Deep Green And Monolithic White LEDs Based On Combination Of Short-Period InGaN/GaN Superlattice And InGaN QWs
}

\author{
A. F. Tsatsulnikov ${ }^{1}$, W. V. Lundin ${ }^{1}$, A. V. Sakharov ${ }^{1}$, E .E. Zavarin ${ }^{1}$, S. O. Usov' ${ }^{2}$, \\ A. E. Nikolaev ${ }^{1}$, N. V. Kryzhanovskaya ${ }^{2}$, A. E. Chernyakov ${ }^{2}$, A. L. Zakgeim ${ }^{2}$, \\ N.A. Cherkashin ${ }^{3}$, M. Hytch ${ }^{3}$ \\ ${ }^{1}$ Ioffe Physical Technical Institute, Russian Academy of Sciences, St. Petersburg, Russia \\ ${ }^{2}$ Submicron Heterostructures for Microelectronics Research \& Engineering Center, Russian Academy of Sciences, \\ St. Petersburg, Russia \\ ${ }^{3}$ CEMES-CNRS-Université de Toulouse, Toulouse, France
}

\begin{abstract}
This work presents the results of the investigation of approaches to the synthesis of the active region of LED with extended optical range. Combination of short-period InGaN/GaN superlattice and InGaN quantum well was applied to extend optical range of emission up to $560 \mathrm{~nm}$. Monolithic white LED structures containing two blue and one green QWs separated by the short-period InGaN/GaN superlattice were grown with external quantum efficiency up to 5-6\%.
\end{abstract}

Keywords: LED, superlattice, quantum well, MOCVD.

PACS: 81.07.Ta, 81.05.Ea, 85.60.Jb, 81.15.Gh

\section{INTRODUCTION}

LEDs with emission wavelength $>530 \mathrm{~nm}$ have poor efficiency due to many problems such as quality of the InGaN layers with high In content, quantum confined Stark effect and Auger processes which also becomes stronger with InGaN composition increase. But development of such LEDs is very important for new generation of multy-color light emitters including smart-white RGB light sources for SSL [1]. In this paper we report significant improvement of deep-green LED properties by modifications of structure design and development of monolithic white LEDs.

\section{EXPERIMENT}

The investigated LED structures were grown in AIX2000HT system with 6 x 2" planetary reactor on (0001) sapphire substrates. Active region of deep green LEDs contains single $3 \mathrm{~nm}$ thick InGaN layer deposited on InGaN/GaN short-period superlattice (SPSL). The SPSL contains 12 periods and was formed by InGaN-conversion technique [2-4]. InGaN QW and SPSL were separated by GaN barrier grown at the same temperature as SPSL. Monolithic white
LED contains in active region one green InGaN QW and two blue QWs separated by the same SPSL. Grown structures were investigated using spectroscopy of photo- and electroluminescence, high-resolution transmission electron microscopy.

\section{RESULTS AND DISCUSSION}

Because recombination of carriers occurs in the last QW, conventional design of the LEDs active region includes top InGaN QW deposited on set of bottom QWs having lower In content or thick low content InGaN layer. To improve structural properties of the active region of deep green LEDs we proposed to replace such structure with the single InGaN QW deposited on top of short-period $1 \mathrm{~nm} \mathrm{InGaN} \mathrm{/} 1 \mathrm{~nm}$ GaN SPSL. It was shown that using of this design of active region leads to the increase of the maximal emission efficiency up to $16 \%$ at $540 \mathrm{~nm}$ and up to $\sim 8 \%$ at $560 \mathrm{~nm}$ (Fig. 1).

For growth of the short period $\mathrm{InGaN} / \mathrm{GaN}$ superlattice method of cycle conversion of the InGaN layer to GaN by applying of growth interruptions in hydroden atmosphere was used. 


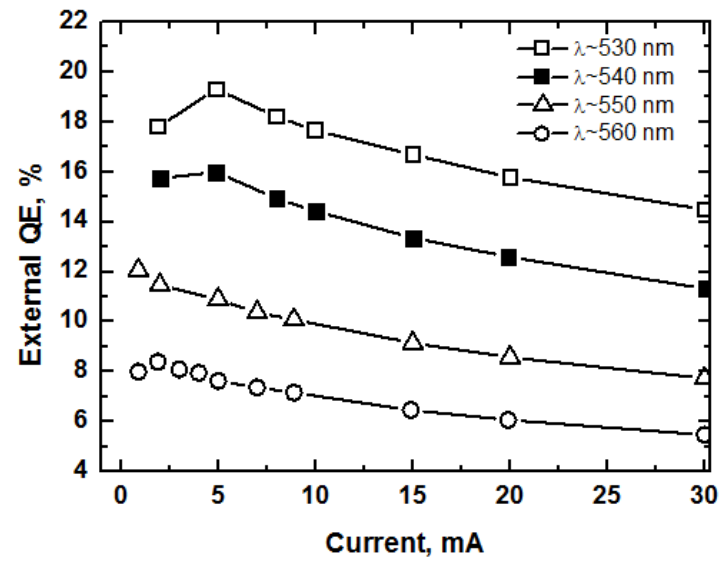

FIGURE 1. Quantum efficiencies of the green LEDs..

Detail investigations of the properies of these SPSL by high resolution transmission electron microscopy (HRTEM), XRD and photoluminescence revealed formation of periodical $\mathrm{InGaN} / \mathrm{GaN}$ structures with rough interfaces and regions with coalescence of neighboring InGaN layers (Fig. 2). These regions are non-correlated in the vertical direction. The coalescence of neighboring InGaN layers results in the dependence of a PL line shape of the SPSL on the number of periods in the $400-470 \mathrm{~nm}$ spectral range. It was shown that such SPSL allows to improve structural properties and carrier transport in the InGaN active region $[2,3]$.

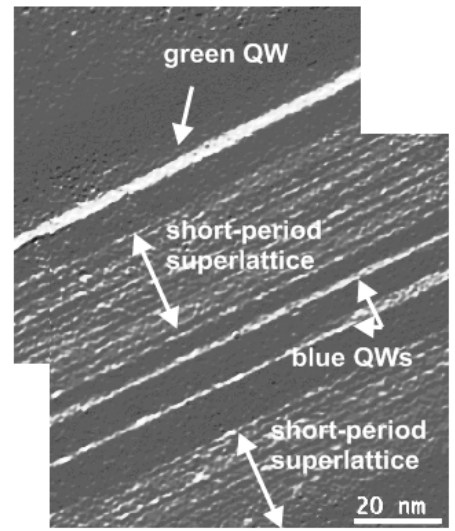

FIGURE 2. HRTEM image of the active region of the monolithic white LED.

The approach based on combination of the SPSL with InGaN QWs was applied for development of effective monolithic white light emitters [5]. This LED contains short period InGaN/GaN superlattice as barrier layer between InGaN QWs emitting in blue and yellow-green ranges (HRTEM image of the active region of the monolithic white LED is shown in the Fig. 2). Optical properties of such structures were studied and it was shown that using of this SPSL allows realization of effective emission from the all active InGaN layers despite on significant (24 nm) distance between blue and green QWs. White light emission with maximal external quantum efficiency of $\sim 5-6 \%$ and CCT in the range of $6000-7600 \mathrm{~K}$ was demonstrated from these LEDs (Fig. 3).

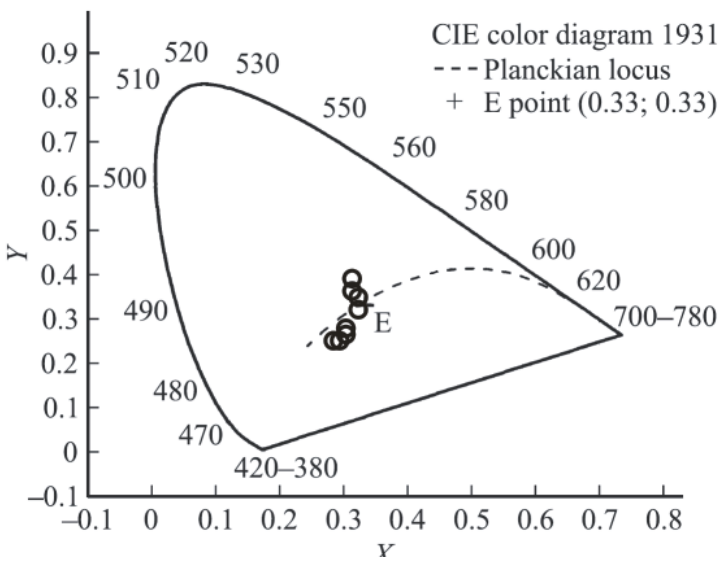

FIGURE 3. CIE color diagram of the monolithic white LED.

\section{ACKNOWLEDGMENTS}

This work was supported by RFBR grants 09-0212449 and 10-02-01044-a.

\section{REFERENCES}

1. A.Zukauskas, M.Shur, R.Gaska. Introduction to SolidState Lighting. J. Wiley \& Sons, NY, 2002.

2. A.F. Tsatsulnikov, W.V. Lundin, A.V. Sakharov, E.E. Zavarin, S.O. Usov, A.E. Nikolaev, N.A. Cherkashin, B.Ya. Ber, D.Yu. Kazantsev, M.N. Mizerov, Hee Seok Park, M. Hytch and F. Hue, Semiconductors, 44 (1), 93 97 (2010).

3. N.V. Kryzhanovskaya, W.V. Lundin, A.E. Nikolaev, A.F. Tsatsul'nikov, A.V. Sakharov, M.M. Pavlov, N.A. Cherkachin, M.J. Hytch, G.A. Valkovsky, M.A. Yagovkina, S.O. Usov, Semiconductors, 44 (6), 857-860 (2010).

4. W.V. Lundin, E.E. Zavarin, M.A. Sinitsyn, A.E. Nikolaev, A.V.Sakharov, A.F. Tsatsulnikov, E.V. Yakovlev, R.A. Talalaev, A.V. Lobanova, A.S. Segal, EWMOVPE 13, Ulm, 7-10 June 2009, Booklet of Extended abstracts, Inv 2.

5. A.F. Tsatsulnikov, W.V. Lundin, A.V. Sakharov, E.E. Zavarin, S.O. Usov, A.E. Nikolaev, N.V.

Kryzhanovskaya, M.A. Sinitsyn, V.S. Sizov, A.L. Zakgeim, M.N. Mizerov, Semiconductors. 44(6), 837-840 (2010) 